\title{
artigo
}

Maia da Silva, N.; Bastos, R.M.; Conceição, V.R.C.; Bastos, A.S.C.; Sales, G.S.; Nogueira Silva, T.;

Promoção da saúde no Brasil na pandemia por COVID-19: concepções e práticas em atenção básica

DOl: https://doi.org/10.36489/saudecoletiva.2020v10i58p4021-4030

\section{Promoção da saúde no Brasil na pandemia por COVID-19: concepções e práticas em atenção básica}

\author{
Health promotion in Brazil in pandemic by COVID - 19: conceptions and practices in basic care \\ Promoción de salud en Brasil en pandemia por COVID -19: concepciones y prácticas en cuidados básicos
}

\begin{abstract}
RESUMO
Objetivo: 0 objetivo deste artigo é analisar, por meio de uma revisão da literatura, a promoção da saúde no brasil em tempos de pandemia por COVID-19, considerando o pensamento dos autores selecionados para dar embasamento ao tema abordado. Método: Tratando-se de um estudo de revisão bibliográfica. Resultados: A promoção da saúde envolve um conjunto de políticas e medidas visando à melhoria das condições de vida e saúde da população e vem sendo discutida desde meados da década de 80, influenciando inúmeras estratégias. Muitos autores enfatizam a promoção da saúde como marco para a melhoria da qualidade de vida da população destacando os fatores condicionantes e determinantes da saúde. Conclusão: A efetivação da promoção da saúde no contexto da ESF em tempos de pandemia por COVID-19 apresenta-se como essencial para caracterizar tal estratégia.
\end{abstract}

DESCRITORES: Promoção da saúde, Estratégia Saúde da Família, Política de Saúde.

\section{ABSTRACT}

Objective: The objective of this article is to analyze, through a literature review, health promotion in Brazil in times of pandemic by COVID-19, considering the thoughts of the selected authors to support the topic addressed. Method: This is a literature review study. Results: Health promotion involves a set of policies and measures aimed at improving the population's living and health conditions and has been discussed since the mid-1980s, influencing numerous strategies. Many authors emphasize health promotion as a milestone for improving the population's quality of life, highlighting the conditioning and determinant factors of health. Conclusion: The effectiveness of health promotion in the context of the FHS in times of pandemic by COVID-19 is essential to characterize this strategy.

DESCRIPTORS: Health Promotion, Family Health Strategy, Health Policy.

\section{RESUMEN}

Objetivo: El objetivo de este artículo es analizar, a través de una revisión de la literatura, la promoción de la salud en Brasil en tiempos de pandemia por COVID-19, considerando los pensamientos de los autores seleccionados para apoyar el tema abordado. Método: Este es un estudio de revisión de la literatura. Resultados: La promoción de la salud involucra un conjunto de políticas y medidas encaminadas a mejorar las condiciones de vida y de salud de la población y ha sido discutida desde mediados de la década de 1980, incidiendo en numerosas estrategias. Numerosos autores destacan la promoción de la salud como un hito para mejorar la calidad de vida de la población, destacando los condicionantes y determinantes de la salud. Conclusión: La efectividad de la promoción de la salud en el contexto de la ESF en tiempos de pandemia por COVID-19 es fundamental para caracterizar esta estrategia.

DESCRIPTORES: Promoción de la Salud, Estrategia de Salud Familiar, Política de Salud.

RECEBIDO EM: 29/08/2020 APROVADO EM: 24/09/2020

\section{Naiara Maia da Silva}

Especialista, Coordenadora da Atenção Básica. Conceição da Feira, BA, Brasil.

ORCID: 0000-0002-2405-0542

\section{Raquel Machado Bastos}

Especialista, Secretária Municipal de Saúde. Conceição da Feira, BA, Brasil.

ORCID: 0000-0002-8119-4649 


\section{Valdirene Rosa da Cruz Conceição}

Especialista, Coordenadora de Saúde do Trabalhador. Conceição da Feira, BA, Brasil.

ORCID: 0000-0001-9932-0706

\section{Andreia de Souza Costa Bastos}

Especialista, Coordenadora do Programa Saúde na Escola, Conceição da Feira, BA, Brasil.

ORCID: 0000-0003-1119-2346

\section{Glauciane da Silva Sales}

Especialista, Coordenadora de Faturamento do Hospital Municipal de Teixeira de Freitas, Teixeira de Freitas, BA, Brasil. ORCID: 0000-0002-5555-1059

\section{Thiago Nogueira Silva}

Mestre, Coordenador de Vigilância em Saúde, Conceição da Feira, BA, Brasil. ORCID: 0000-0002-8666-8698

\section{INTRODUÇÂO}

A promoção da saúde pode ser entendida, como um conjunto de medidas capazes de atuar sobre os determinantes da saúde, aumentando o bem estar geral, porém não restringindo a saúde à ausência de doenças. Perpassam os serviços clínicos assistenciais envolvendo ações intersetoriais no âmbito da educação, saneamento básico, habitação, renda, trabalho, alimentação, acesso a bens e serviços essenciais, lazer, entre outros ${ }^{1}$.

Com isso, a Atenção Básica torna-se um dos principais instrumentos a serem utilizados como veículo para a Promoção da Saúde, atuando como mediadora entre as famílias e a implantação das políticas de saúde, estabelecendo atividades de prevenção, promoção, recuperação e reabilitação da saúde da saúde dos cidadãos ${ }^{1}$.

De acordo com a Organização Mundial da Saúde, a promoção da saúde possibilita que indivíduos ampliem o controle sobre os determinantes da saúde ${ }^{2}$. Nesse sentido, a necessidade que haja mudanças nos modos e condições de vida e o seu papel como uma estratégia de mediação entre as pessoas e o meio ambiente ${ }^{3}$.

Nos últimos 25 anos o mundo reconheceu a importância da Promoção da Saúde com a realização de importantes Conferências Internacionais sobre Promoção da Saúde. Documentos importantes como: o relato das emissões enviadas à China em 1973 e 1974, o Relatório Lalonde no Canadá em 1974, a Declaração de Alma
Ata em 1978 e Conferências Internacionais sobre Promoção da Saúde realizadas em Ottawa em 1986, Adelaide em 1988, Sundsvall 1991, Jacarta em 1997, Bangkok 2005, desenvolveram as bases políticas e conceituais da promoção da saúde. Bem como, na América Latina, a Conferência de Santafé de Bogotá em 1992, a V Conferência Internacional no México em 2000 e a Conferência Mundial pelo Meio ambiente, conhecida como ECO 92, também fortaleceram os ideais de promoção da saúde trazendo o tema para o referido continente ${ }^{4}$.

Na década de 90, os olhares para a promoção da saúde passam a crescer no pensamento sanitário brasileiro com às propostas de vigilância à saúde e estratégias como Cidades Saudáveis e Saúde da Família. Assim, surgiu no Brasil, em 2006, a Política Nacional de Promoção da Saúde, que propõe intervenções em problemas e necessidades de saúde visando reduzir riscos para a saúde da população $0^{5,6}$.

Assim, o objetivo deste artigo é analisar, por meio de uma revisão da literatura, a promoção da saúde no brasil em tempos de pandemia por COVID-19, considerando o pensamento dos autores selecionados para dar embasamento ao tema abordado.

\section{MÉTODO}

A metodologia utilizada para este artigo foi à revisão bibliográfica que se refere às atividades envolvidas na busca de informações sobre um tópico e na elaboração de um quadro abrangente da situação de informações ${ }^{23}$.

Para esta revisão, foi realizada busca em artigos de revistas científicas disponíveis nas seguintes bases de dados: Medical Literature Analysis and Retrieval System Online (MEDLINE), Literatura Latino-Americana e do Caribe em Ciências da Saúde (LILACS) e na biblioteca virtual Scientific Electronic Library Online (SciELO).

A pesquisa dos artigos utilizados foi efetuada do início ao final de Junho de 2020. Foram estabelecidos como critérios de inclusão: artigos científicos completos nos idiomas português, inglês e espanhol; disponíveis para consulta e publicados nos últimos 5 anos e que pudessem trazer elucidação para a questão norteadora.

Para a categorização dos estudos, as informações extraídas destas publicações foram organizadas. Posteriormente, essas informações foram dispostas em parágrafos de acordo com os principais resultados do artigo. Também foram consideradas as metodologias utilizadas pelos autores e as conclusões alcançadas.

\section{RESULTADOS E DISCUSSÃO}

Para uma melhor organização da discussão dos dados obtidos, os resultados foram divididos em tópicos específicos, com agrupamento dos mesmo conforme a temática relativa as informações, como segue: 


\section{Promoção da Saúde no Brasil}

Os questionamentos sobre a promoção da saúde foram introduzidos no Brasil em meados dos anos 80 , em consonância com os debates da Reforma Sanitária, recebendo destaque na $8^{a}$ Conferência Nacional da Saúde em 1986. Nessa conferência houve um consenso de que para o setor saúde no Brasil, não era suficiente uma mera reforma administrativa e financeira, mais sim uma mudança em todo arcabouço jurídico institucional vigente que contemplasse a ampliação do conceito de saúde ${ }^{1}$.

As constatações desses autores vêm ao encontro da ideia de que ainda que os conceitos e objetivos para a sociedade brasileira, da $8^{a}$ Conferência Nacional eram semelhantes aos propostos em Ottawa, na $1{ }^{a}$ Conferência Global sobre Promoção da Saúde ${ }^{7}$. Dessa maneira, a Carta de Ottawa propõe uma concepção positiva de saúde, na qual foi formulada uma base ideológica para a promoção da saúde, um recurso do dia a dia, não um objetivo da vida, ressaltando a sua importância para uma vida social e economicamente produtiva ${ }^{4}$.

Com isso, a promoção da saúde é um campo teórico - prático - político que em sua composição com os conceitos e posições do Movimento de Reforma Sanitária brasileira delineia-se como uma política que deve percorrer o conjunto de ações e projetos em saúde, apresentando-se em todos os níveis de complexidade da gestão e atenção do sistema de saúde [...] que coloque os sujeitos - usuários e profissionais de saúde -, como protagonistas na organização do processo produtivo em saúde, entendendo que aí se produz saúde, sujeitos e mundo 8 .

Com isso, no Brasil, o ideário da promoção da saúde apresenta-se como influenciador da proposta de Vigilância à Saúde, sustenta o Projeto de Cidades Saudáveis, influencia práticas de educação à saúde e projetos de reorganização da rede básica, hoje vinculados à Estratégia de Saúde da Família?.

A Política Nacional de Promoção da Saúde propõe intervenções em eixos temáticos, modos de viver, condições de trabalho, habitação, ambiente, educação, lazer,
Com isso, a promoção da saúde propõe uma atuação que reverta situações como: falta de oportunidade de escolha e controle dos indivíduos sobre situações de vida, para que a equidade seja alcançada, ou seja, possam ser criadas oportunidades para a justiça social, em que diferenças biológicas

e a livre escolha sejam respeitadas, e asseguradas condições

\section{mínimas para uma}

\section{sobrevivência digna.}

cultura, acesso a bens e serviços essenciais ${ }^{5}$.

A Política Nacional de Promoção da Saúde, trata-se então de uma estratégia de produção de saúde, ou seja, um modo de pensar e de operar articulado às demais políticas e tecnologias desenvolvidas no sistema de saúde brasileiro.

\section{Promoção da Saúde: Conceitos e Sig- nificados}

Promoção da saúde é o nome dado ao processo de capacitação da comunidade para atuar na melhoria de sua qualidade de vida e saúde, incluindo uma maior participação no controle deste processo ${ }^{10}$. É uma estratégia que tem como objetivo principal melhorar a qualidade de vida da população e consequentemente as condições de saúde. Para isso, existe a necessidade de um trabalho articulado entre distintos setores visando interferir diretamente nos condicionantes e determinantes da saúde, bem como, trabalhar entre os indivíduos a responsabilidade que cada um tem com a sua própria saúde.

A carta de Ottawa, resultado da $1^{\text {a }}$ Conferência Internacional sobre Promoção da Saúde realizada em 1986 no Canadá, propôs uma concepção positiva de saúde, enfatizando que a promoção da saúde não é responsabilidade exclusiva do setor saúde, pois envolve um estilo de vida saudável e um bem estar global. Ainda foram consideradas condições fundamentais para a existência de saúde: ter paz, habitação, educação, alimentação, renda, ecossistema estável, recursos sustentáveis, justiça social e equidade ${ }^{10}$.

Em consonância com este estudo, é declarado que caracterizar a promoção da saúde sustenta-se no entendimento que a saúde é produto de um conjunto de fatores relacionados com a qualidade de vida, incluindo boas condições de trabalho, oportunidade de educação ao longo da vida, ambiente físico limpo, apoio social para famílias e indivíduos e possibilidade de cuidado com a saúde ${ }^{11}$.

Com isso, a promoção da saúde propõe uma atuação que reverta situações como: falta de oportunidade de escolha e controle dos indivíduos sobre situações de vida, para que a equidade seja alcançada, ou seja, possam ser criadas oportunidades para a justiça social, em que diferenças biológicas e a livre escolha sejam respeitadas, e asseguradas condições mínimas para uma sobrevivência digna ${ }^{12}$. 
Nisso, uma vez que tem suas ações e políticas dirigidas aos determinantes da saúde, a promoção da saúde requer a cooperação entre os diferentes setores envolvidos e a articulação de suas ações: legislação, sistema tributário, medidas fiscais, educação, habitação, serviços sociais, cuidados primários em saúde, planejamento urbano, entre outros ${ }^{13}$.

Dessa forma, a promoção da saúde defini-se de maneira mais ampla que prevenção, pois refere-se a medidas que não se dirigem a uma determinada doença ou desordem servindo para aumentar a saúde e o bem estar gerais ${ }^{14,15}$.

Assim, assumindo a relação da saúde com a política, economia, meio-ambiente e os fatores sócio-culturais (além dos biológicos), atribui-se a promoção da saúde o dever de permitir que tais fatores sejam favoráveis à saúde.

A importância do envolvimento de diferentes atores para a promoção da saúde foi destacada desde 1978, na Declaração de Alma Ata, que colocou a saúde como direito humano fundamental e o mais alto nível de saúde, como uma meta social mundial não só do setor saúde, como também social e econômico. Reforçando esta linha, em 1988, na Austrália, na Conferência de Adelaide, segunda Conferência Internacional sobre Promoção da Saúde foi instituída uma nova orientação para as políticas de saúde, dando ênfase ao envolvimento das pessoas, cooperação de vários setores da sociedade e a criação dos cuidados primários de saúde ${ }^{16,17}$.

\section{Promoção da Saúde na Atenção Bási- ca em Tempos de Pandemia por Co- vid-19}

A Atenção Básica/Saúde da Família é organizada por meio de trabalho interdisciplinar em equipe, mediante a responsabilização de Equipes de Saúde da Família num dado território, área de abrangência de uma população adscrita. Trabalha com foco nas famílias, por intermédio de vínculos estabelecidos, desenvolvendo ações de promoção da saúde, prevenção de doenças, recuperação e reabilitação da saúde. De acordo com a Portaria 648, de 28 de março de 2006 que aprova a política $\mathrm{Na}$ cional da Atenção Básica, a ESF visa a reorganização da Atenção Básica no país, de acordo com os preceitos do SUS ${ }^{18}$.

A Organização Mundial da Saúde (OMS) declarou Em 12 de março de 2020, como pandemia a doença causada pelo novo coronavírus (SARS-CoV-2). A infecção (COVID-19), além de ser mais transmissível, tem letalidade estimada em cerca de 14 vezes a da influenza. O alto índice de contágio, com cada indivíduo infectando de 2 a 3 pessoas, em média, causa a expansão da epidemia em progressão geométrica ${ }^{19}$.

No Brasil, a priorização da Atenção Primaria a Saúde - APS, com a expansão da Estratégia Saúde da Família e o fortalecimento de todos os seus atributos, com ênfase nos chamados derivados (competência cultural, orientação familiar e comunitária) é crucial no combate ao COVID-19 em todo o território nacional e a APS tem papel preponderante para garantir o acesso a cuidados de saúde e agir sobre os determinantes de saúde frente a um desafio emergente ${ }^{20}$.

A resposta nacional ao COVID-19 passa pela APS com as Equipes de Saúde da Família no papel de coordenação e comunicação do cuidado, desenvolvendo ações para a população e comunidades, buscando a diminuição drástica das pessoas que seriam encaminhadas, desnecessariamente, aos prontos-socorros e hospitais públicos e privados. Além disso, é fundamental que a ESF reforce as estratégias de prevenção para a COVID-19 e, ao mesmo tempo, mantenha o acompanhamento longitudinal das pessoas sob cuidado ${ }^{21}$.

As ações da Atenção Básica, em especial da Estratégia de Saúde da Família, num cenário de escassez de exames diagnósticos e de leitos de tratamento intensivo mostram-se essenciais, tais como:

- Busca ativa de sintomáticos respiratórios; Representa a tática inicial para a detecção precoce de infectados recentes e, quando incorporada ao processo de trabalho das equipes, promovendo o diagnóstico prévio da doença, e evitando a subnotificação dos $\operatorname{casos}^{22}$;
- Monitoramento dos casos confirmados no território; Quando um caso suspeito de COVID-19 é identificado e notificado inicia-se o monitoramento. Nesse contexto, os casos passam a ser monitorados via telefone até a alta e onde, no diálogo, observa-se a sintomatologia, a evolução clínica e é verificado se o isolamento está adequado e se alguém da família manifestou sintomas da doença e, quando necessário, a equipe da ESF se desloca até o lar para uma melhor avaliação e monitoramento in loco $^{22}$.

- Isolamento social e ESF; O isolamento social horizontal tem sido uma importante estratégia de controle mundial da COVID-19 para a redução do crescimento exponencial de casos. O distanciamento social horizontal é necessário para a postergação do pico epidêmico, reduzindo o impacto desta por demanda assistencial e dá tempo para que os gestores organizem a rede assistencial, evitando consequências na saúde da população ${ }^{22}$.

- Coordenação do cuidado às famílias; A ESF assume papel estratégico no Sistema Único de Saúde (SUS), na coordenação do cuidado no território sanitário e no ordenamento da RAS, com o intuito de garantir a promoção da saúde, a prevenção da doença, assim como, a identificação precoce, monitoramento, assistência e reabilitação dos doentes, acompanhando os usuários do território sanitário e, ao identificar a necessidade de compartilhamento do cuidado em rede com os demais serviços, dialogam com a atenção especializada para a realização de exames de imagem e com os hospitais para o processo de internação ${ }^{22}$.

Mediante desses aspectos, torna-se necessário evidenciar a promoção da saúde no Brasil em especial, na pandemia por COVID-19.

\section{CONCLUSÃO}

Considerando os estudos realizados e os objetivos levantados concluímos que a Promoção da Saúde é uma estratégia que quando realizada de acordo com o que é preconizado pelo Ministério da Saúde, 


\section{artigo}

Maia da Silva, N.; Bastos, R.M.; Conceição, V.R.C.; Bastos, A.S.C.; Sales, G.S.; Nogueira Silva, T.

Promoção da saúde no Brasil na pandemia por COVID-19: concepções e práticas em atenção básica

traz vantagens para a população assistida ao serem analisados os aspectos inerentes a promoção da saúde no Brasil em tempos de pandemia por COVID-19.

A análise das origens e concepções da promoção da saúde no Brasil proporcionaram ampliar o conhecimento de quando surgiram os primeiros questionamentos sobre esta temática no país o que possibilitou inúmeras discussões e reflexões de diferentes autores.
Entendendo a promoção da saúde como um fator considerável que possibilita a visibilidade das causas determinantes das condições de saúde da população podemos entendê-la como fundamental para a melhoria da qualidade de vida dos beneficiados pelas ações e atividades dentro do contexto da referida estratégia.

Ao darmos ênfase à identificação da promoção da saúde no âmbito da Estratégia de Saúde da Família foi destaque a importância desta ferramenta para buscar a melhoria das condições de vida e saúde da população, principalmente no que se refere a necessidade de mostrar para os indivíduos a responsabilidade que cada um tem com a sua saúde e desenvolver processos educativos voltados para a melhoria do autocuidado dos mesmos, um dos pontos principais a ser trabalhado quando se fala em promover saúde na Atenção Básica.

\section{REFERÊNCIAS}

1. Lopes AMP. Promoção da saúde no processo de democratização brasileiro: biopolíticas e constituição de sujeitos da saúde. Fractal, Rev. Psicol. 2019;31(3):283-291.

2. Silva JBJ. Promoção da saúde: ação necessária e urgente nas Américas. Ciênc. saúde coletiva. 2019;24(11):3994.

3. Abreu MF, Melo EM e Silveira AM. Contribuições do Planejamento Participativo Regionalizado (PPR) para a ampliação da cidadania e promoção da saúde. Saúde em Debate [online]. 2018;42(spe4):107-119.

4. Malta DC et al. O SUS e a Política Nacional de Promoção da Saúde: perspectiva resultados, avanços e desafios em tempos de crise. Ciênc. saúde coletiva. 2018;23(6): 1799-1809.

5. Dias MSA et al. Política Nacional de Promoção da Saúde: um estudo de avaliabilidade em uma região de saúde no Brasil. Ciênc. saúde coletiva. 2018;23(1):103-114.

6. Hartz ZMA. Cuidados primários, avaliação e ações intersetoriais em promoção da saúde. Ciênc. saúde coletiva. 2017;22(3):687-688.

7. Minayo MCS. Políticas, tecnologias e práticas em promoção da saúde. Ciênc. saúde coletiva. 2017;22(12):4151-4152.

8. Penido CMF, Romagnoli RC. Apontamentos sobre a clínica da autonomia na promoção da saúde. Psicol. Soc. 2018;30:e173615.

9. Oliveira JLM et al. Os desafios do saneamento como promoção da saúde da população brasileira. Saúde em Debate [online]. 2019;43(especial 3):4-5.

10. OPAS. Organização Pan-americana de Saúde Carta de Ottawa: primeira conferência internacional sobre promoção da saúde, Ottawa,1986. Disponivel em: <http://www.opas.org.br/ promocao/uploadArq/Ottawa.pdf> Acesso em: 10 jul. 2020.

11. Dias LF et al . Promoção da Saúde: Coerência nas Estratégias de Ensino-Aprendizagem. Rev. bras. educ. med. 2019;43(1),supl.1:641-651.

12. Ramos JFC et al . Pesquisa participativa e as estratégias de promoção da saúde integral da criança no Sistema Único de Saúde (SUS). Interface (Botucatu). 2018;22(67):1077-1089.

13. Oliveira RTQ et al . Matriz de avaliação de programas de pro- moção da saúde em territórios de vulnerabilidade social. Ciênc. saúde coletiva. 2017;22(12):3915-3932.

14. Madeira FB et al. Estilos de vida, habitus e promoção da saúde: algumas aproximações11Apoio financeiro: Fundação de Amparo à Pesquisa do Estado do Piauí (Fapepi).. Saúde e Sociedade [online]. 2018; 27(1):106-115.

15. Prado, Nilia Maria de Brito Lima e Santos, Adriano Maia dos Promoção da saúde na Atenção Primária à Saúde: sistematização de desafios e estratégias intersetoriais. Saúde em Debate [online]. 2018;42(spe1):379-395.

16. OPAS. Declaração de Alma Ata: Conferência internacional sobre cuidados primários de saúde,URSS, 1978. Disponível em:< http://www.opas.org.br/coletiva/uploadArq/Alma-Ata.pdf>. Acesso em: 10 jul. 2020.

17. OPAS. Conferência de Adelaide: segunda conferência internacional sobre promoção da saúde, Austrália,1988. Disponível em: <http://www.opas.org.br/promocao/uploadArq/Adelaide. pdf>. Acesso em: 12. jul.2011.

18. Farias JM, Minghelli LC, Soratto J. Promoção da saúde: discursos e concepções na atenção primária à saúde. Cad. saúde colet. 2020. Ahead of Print:1-9.

19. WHO - World Health Organization. Report of the WHO-China Joint Mission on Coronavirus Disease 2019 (COVID-19). Genebra:WHO, 2020.

20. Daumas, Regina Paiva et al. O papel da atenção primária na rede de atenção à saúde no Brasil: limites e possibilidades no enfrentamento da COVID-19. Cadernos de Saúde Pública [online]. 2020;36(6):1-7.

21. Harzheim E et al. Ações federais para apoio e fortalecimento local no combate ao COVID-19: a Atenção Primária à Saúde (APS) no assento do condutor. Ciência \& Saúde Coletiva [online]. 2020;25(suppl 1):2493-2497.

22. Ximenes N, Francisco RG et al. Coordenação do cuidado, vigilância e monitoramento de casos da COVID-19 na Atenção Primária à Saúde. Enfermagem em Foco, [S.I.]. 2020;11(1).

23. GIL, A.C. Como elaborar projetos de pesquisa. 4. Ed. São Paulo: Atlas, 2002. 